\title{
El modelado físico del espacio de diseño concurrente como recurso didáctico para el análisis, exploración y mediación entre lo abstracto y concreto.
}

\author{
Briede W., Juan Carlos ${ }^{\text {a }}$, Leal F., Isabel Margarita ${ }^{\mathrm{b}}$ \& Pérez V., Cristhian ${ }^{\mathrm{c}}$ \\ ${ }^{a}$ Diseñador Industrial. Departamento de Arte y Tecnologías del Diseño, Universidad del Bío-Bío, Chile. \\ jbriede@ubiobio.cl \\ bDiseñadora Gráfica. Departamento de Arte y Tecnologías del Diseño, Unviersidad del Bío-Bío, Chile. \\ ileal@ubiobio.cl \\ 'Psicólogo. Departamento de Educación Médica, Universidad de Concepción, Chile.cperezv@udec.cl
}

\begin{abstract}
Resumen
Las metodologías de diseño de productos orientadas a potenciar la creatividad hacia la innovación requieren apoyarse en métodos y herramientas para pasar de la etapa divergente de análisis a una convergente de sintesis que se presenta al momento de abordar el diseño conceptual. El modelo simplificado para abordar el diseño conceptual planteado por Hernandis \& Briede (2009), permite modelar el espacio del diseño del producto desde una perspectiva teórica, mapeando e integrando los requerimientos y condicionantes a tener en cuenta en esta etapa de desarrollo y servir como guía de referencia, a través de un esquema visual integral, que sirve como guía o mapa teórico al momento de configurar y caracterizar la propuesta de diseño conceptual. Este proceso, constituye un ejercicio cognitivo, que requiere un alto grado de abstracción que muchas veces dificulta y desalienta a los estudiantes, ya que no son capaces de alcanzar a identificar sus ventajas y mas aún conectarlas con la aplicación real.
\end{abstract}

Como estudio de caso, en el diseño y desarrollo de productos abordado por el Taller de Diseño Centrado en el Usuario [DCU] de tercer año de la carrera de diseño industrial de la Universidad del Bío-Bío, se ha implementado la metodología sistémica a modo de guiar el proceso y la toma de decisiones en el modelado conceptual. Para lograrlo se aplicó la secuencia metodológica de Hernandis \& Briede (2009), con la cual, a la hora de modelar el espacio de diseño, los alumnos además de esquematizarlo en una lámina, lo han maquetado tridimensionalmente. Este ejercicio permitió reforzar el proceso de análisis y evidenciar la tensión entre la representación teórica y la representación física gracias al tiempo de construcción y a la representación de las cualidades geométricamente. Esto permitió a los alumnos entender la conexión entre el modelado teórico y su aplicación en el diseño final, la desagregación geométrica de volumen, superficie y límite de contorno y su traducción a la dimensión concreta y paramétrica del producto. El modelo fisico del espacio del diseño puede estimular y apoyar la exploración de alternativas como recurso mediador de los atributos teóricos y su resolución tangible en el plano del producto. Se constata que incluir esta etapa facilita el "modelado del espacio del diseño" así como la gestión e integración de variables y atributos del producto antes de generar la propuesta de diseño. 
El modelado físico del espacio de diseño concurrente como recurso didáctico para el análisis, exploración y mediación entre lo abstracto y concreto.

Palabras Clave: Diseño Conceptual, Modelado Concurrente, Maqueta, Espacio de Diseño, Educación en Diseño.

\begin{abstract}
Product design methodologies supporting creativity and innovation require tools and methods that bridge divergent stages of analysis with concurrent stages of synthesis during conceptual design. The simplified model for conceptual design presented by Hernandis \& Briede (2009) works from a theoretical perspective, allowing designers to model the design space of a given product by mapping and integrating diverse requirements and conditions. The resulting reference guide or theoretical map presents a visual, integrated framework for configuring and characterizing the conceptual design proposal. This is, above all, a cognitive exercise. It requires a high level of abstraction, which may challenge and discourge design students who are unable to identity the benefits of the task at hand and apply it to real-world situations.
\end{abstract}

This paper presents a case study of the User-centred Design (UCD) Workshop for thirdyear industrial design students at the Universidad del Bio-Bio. A systematic methodology for product design and development was implemented in order to guide the process of design and decision-making during conceptual modelling. Specifically, the sequential methodology proposed by Hernandis \& Briede (2009) was applied. Here, students were asked to outline the design space on a sheet of paper and then create a three-dimensional model. This exercise ultimately strengthened students' ability to analyse and articulate tensions between the theoretical representation and the physical representation of a product. This was aided by the time allotted for construction and the task of geometric modelling. Overall, the activity provided students with a greater understanding of the relationship between theoretical modelling and its application to design, the geometric disaggregation of volume, surface area and contour limits, and its application to the concrete dimensions and parameters of a product. Physical modelling of the design space may help to encourage and support exploration of alternative approaches to design, thereby bridging the gap between theoretical product attributes and the final, tangible product solution. Results confirm that this stage enables the "modelling of the design space" as well as the development and integration of product attributes and other variables prior to developing a design proposal.

Keywords: Conceptual Design, Concurrent Modelling, Model, Design Space, Design Education. 


\section{Introducción}

La relación entre la educación superior y el mercado laboral no es evidente en nuestro contexto, por lo que los alumnos en su proceso de enseñanza aprendizaje no desarrollan las competencias que le permitan insertarse de manera efectiva en el campo laboral y reconocer las particularidades de su entorno. En el caso del diseño, la enseñanza de éste se ha basado tradicionalmente a través de una metodología proyectual (Mabardi 2012), donde la reflexión durante la práctica (Schon,1983) ocurre en un contexto de aprendizaje experiencial.

Por otro lado, el trabajo en proyectos ha sido ampliamente utilizado en estos programas formativos para ayudar a los alumnos a integrar, aplicar y expandir los conocimientos adquiridos en clases teóricas en su curriculum (Dym et al., 2005).

Por lo anterior, la carrera de Diseño Industrial en la Universidad del Bío-Bío en Chile, ha incorporado como estrategia en su proceso formativo el aprendizaje a partir del desarrollo de proyectos con encargos definidos, a fin de promover la formación de las competencias esperadas en el programa de estudio (Ballerini y otros. 2009) con una combinación de las habilidades, y del conocimiento necesario para realizar una asignación particular (SDE, 2001). Esto permite construir nuevo conocimiento, para satisfacer el encargo asignado (Voorhees, 2001; Walter, 2000).

El aprendizaje por proyectos es coincidente con el enfoque de aprendizaje por competencias, que busca facilitar la inserción de los estudiantes en el mundo laboral, permitiéndoles experimentar, a lo largo de los cinco años de carrera, diversos niveles y tipos de intervenciones similares a las del mundo laboral, avanzando por diversos énfasis (observación, producción, usuario, negocio), además de conocer y desarrollar la capacidad de interacción social desde su profesión, permitiéndoles desarrollar las competencias para responder a las nuevas necesidades del entorno (Barberá et al., 2008).

\section{Contextualización metodológica}

El diseño centrado en el usuario, ubica al ser humano como centro, inicio y fin del proceso de diseño [17]. En contexto específicos, como el diseño de las aplicaciones web, considera al usuario un sujeto clave al momento de evaluar, sugerir y hasta diseñar parte de las aplicaciones [18]. Si lo llevamos al diseño de productos existen variados métodos para registrar, sistematizar y utilizar la voz del usuario [19].

El presente trabajo documenta parte de la experiencia, específicamente el diagnóstico, en el Taller "Diseño Centrado en el Usuario" [DCU], parte del proceso formativo de la carrera de Diseño Industrial de la Universidad del Bío Bío, cuyo enfoque metodológico y apuesta busca responder a las características específicas contexto y asumir el rol social del diseño, explorando posibles áreas de desempeño a partir del estudio de comunidades vulnerables y necesitadas (Briede et al. 2011). En este taller se desarrolla la metodología DCU, cuyo énfasis es diseñar un producto, basándose en requerimientos y necesidades de un usuario real en su contexto igualmente real (Lim et al., 2012). Para ello se implementa un enfoque participativo (referencia) que busca integrar activamente al individuo en cuestión, durante el proceso de diseño cambiando del paradigma de diseñar "para la gente" a diseñar "con la gente" .

Se busca abordar una temática altamente relevante para la comunidad vulnerable y que es solicitada por los miembros de ésta. En la propuesta de diseño debe atender a la problemática social presente que emerge, con una mirada contextualizadora que considere el contexto a nivel país y regional, abordando la estrategia y enfoque de diseño inclusivo (Coleman et al., 2007), a fin de considerar y ser capaz de trabajar con contextos en riesgo social de nuestro país.. 
En la experiencia que se documenta en este estudio, es el "emprendimiento callejero" la temática y contexto de estudio abordado, enfocándose en el estudio del fenómeno cada vez en aumento de vendedores ambulantes, no convencionales que buscan acceder a un ingreso, vendiendo una diversidad de productos manufacturados, tales como artesanías, alimentos, joyas, etc. Estos trabajadores, son parte del grupo de trabajadores que pertenecen a la economía informal, que se caracteriza por carecer de contrato, dedicarse a actividades de subsistencia y carecer de protecciones jurídicas y reglamentarias, lo que los convierte en trabajadores de alta vulnerabilidad (Moyano, Castillo y Lizana, 2008)

El objetivo principal de este taller fue aplicar la metodología de diseño centrada en el usuario para diseñar un producto que respondiera a las necesidades de este grupo de trabajadores, contando como objetivos específicos: 1) Comprender y aplicar métodos DCU en el diseño de un producto, 2) Integrar al usuario en el desarrollo del proyecto tanto en las de definición del problema como en el proceso de diseño como proceso de ideación, selección y co-creación, y 3) Comprender, valorar y vivenciar el rol social del diseño ejerciéndolo con responsabilidad social.

\section{Material y Método}

El presente trabajo documenta los resultados de la eplicación del enfoque sistémico en el Taller de Diseño Centrado en el Usuario [DCU] durante la etapa de modelado conceptual. La investigación documenta la implementación de este enfoque durante la etapa de diseño conceptual del taller [DCU] (Briede et al., 2014). En este, se consideró el desarrollo de modelos conceptuales para el análisis desagregado del producto en los subsistemas fundamentals de forma, función y ergonomía (Hernandis y Briede, 2009). En la siguiente tabla se puede observar el momento, dentro de la secuencia de actividades del taller, en que se aplicó la metodología.

Tabla 1. Secuencia de Etapas abordadas en el taller. Fuente: Elaboración Propia

\begin{tabular}{|l|l|}
\hline Etapa & Métodos y Herramientas \\
\hline Inmersión & Observación \\
\hline Invitación & Entrevista informal \\
\hline Observación del Contexto & $\begin{array}{l}\text { Observación /Perfil de Usuario. } \\
\text { Secuencia Diaria. }\end{array}$ \\
\hline Enfocar Problema & Card Sorting/Jerarquización \\
\hline Diseño Conceptual & $\begin{array}{l}\text { Propuesta Conceptual } \\
\text { Métodología Sistémica }\end{array}$ \\
\hline Validación y Testeo & $\begin{array}{l}\text { Prototipo, Check list, } \\
\text { Entrevistas. }\end{array}$ \\
\hline
\end{tabular}

\subsection{Productos esperados de la actividad:}

El taller [DCU] del año 2013 abordó la problemática del microemprendimiento callejero como foco de estudio. En dicha occasion el taller se dividió en grupos formados por 3 a 4 estudiantes y así poder abordar las etapas del proyecto en cuestión. Para ilustrar la aplicación de las fases claves del enfoque 
propuesto se utilizará el proyecto desarrollado por las alumnas Maureen Muñoz, Carmen Pereira y Melissa Quezada.

Tema: Implementación objetual para apoyo de microemprendimientos.

Caso: Exhibición de ponchos en paredes de material ligero.

Propuesta Conceptual: Exhibidor de sobreposición radial para el almacenaje colgado de ponchos en tiendas de material ligero.

Objetivo General: Mejorar la exhibición de ponchos en tiendas de material ligero.

Objetivos Específicos:

- Generar orden en el almacenamiento de ponchos.

- Mejorar la visualización de cada poncho.

- Lograr que la exhibición de los ponchos sea completa, de tal modo que salga del exhibidor sólo al momento de prueba.

Estudio del Contexto y Observaciones:

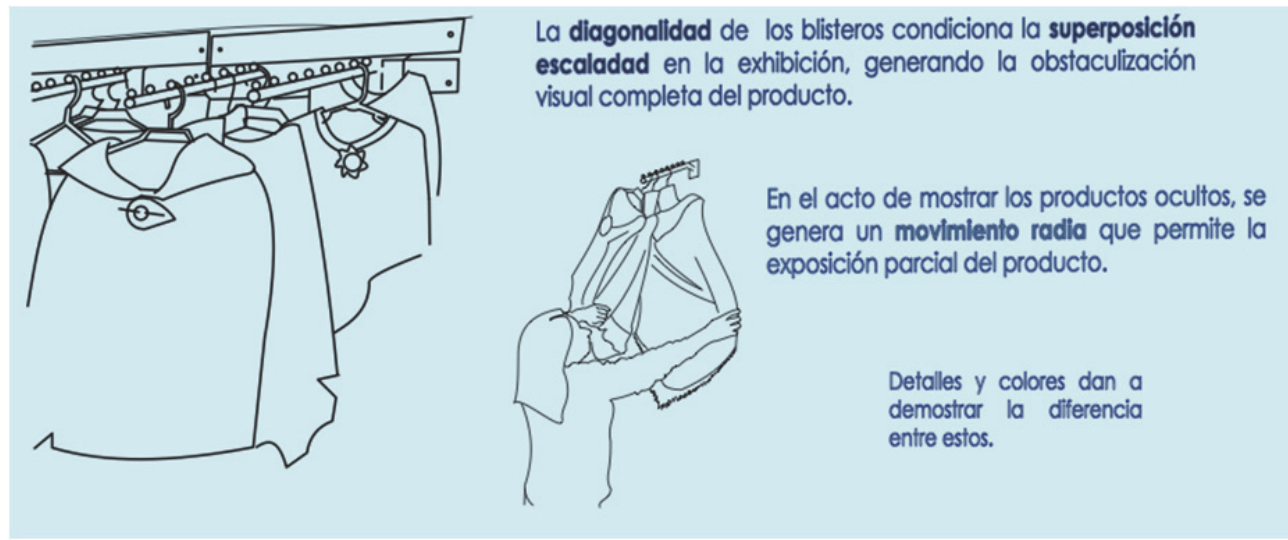

Fig. 1 Observaciones de Contexto Proyecto Colgador. Fuente: Maureen Muñoz,Carmen Pereira y Melissa Quezada (2013).

La realización de la etapa de diseño conceptual considerando el enfoque sistémico, implicaba la realización de los siguientes productos:

Análisis del Estado del Arte: se busca realizar un estudio de mercado respecto a los productos y tipologías como colgadores, tipo de prendas, ponchos, etc.

A través de una matriz que considera evaluar los aspectos atingentes a la forma, función y ergonomía pero de forma desagregada, considerando además por cada uno de ellos las ventajas y desventajas que pueden presentar. 
El modelado físico del espacio de diseño concurrente como recurso didáctico para el análisis, exploración y mediación entre lo abstracto y concreto.

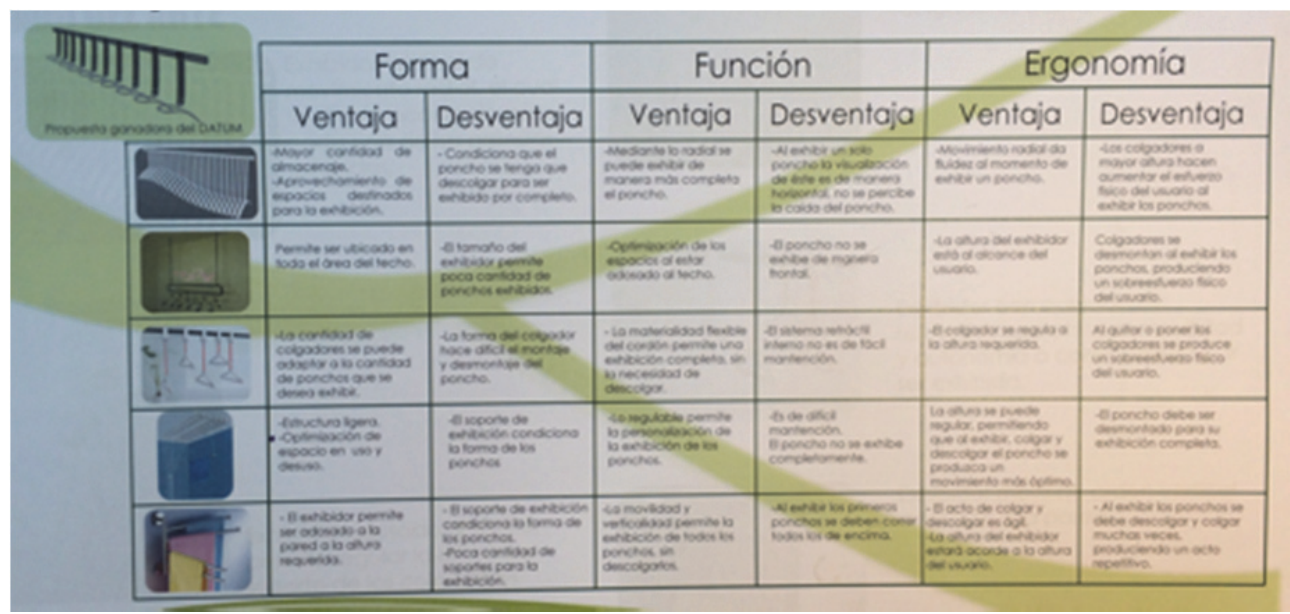

Fig. 2 Análisis del estado del arte. Fuente: Maureen Muñoz,Carmen Pereira y Melissa Quezada (2013).

Ajuste de propuesta conceptual (opcional): En base al análisis del estado del arte y los productos existentes se ajusta la propuesta conceptual si llegara a ser necesario para que sea coherente y que constituya un aporte y novedad al contexto de la problemática.

Definición de modelos conceptuales. Considerando la propuesta conceptual y los atributos seleccionados del estado del arte se procede a definir los atributos y características del producto desagregadamente en forma, función y ergonomía y luego asociarlos a tres tipologías de representaciones geométricas:Volumenes, Superficies y Limites de Contorno.

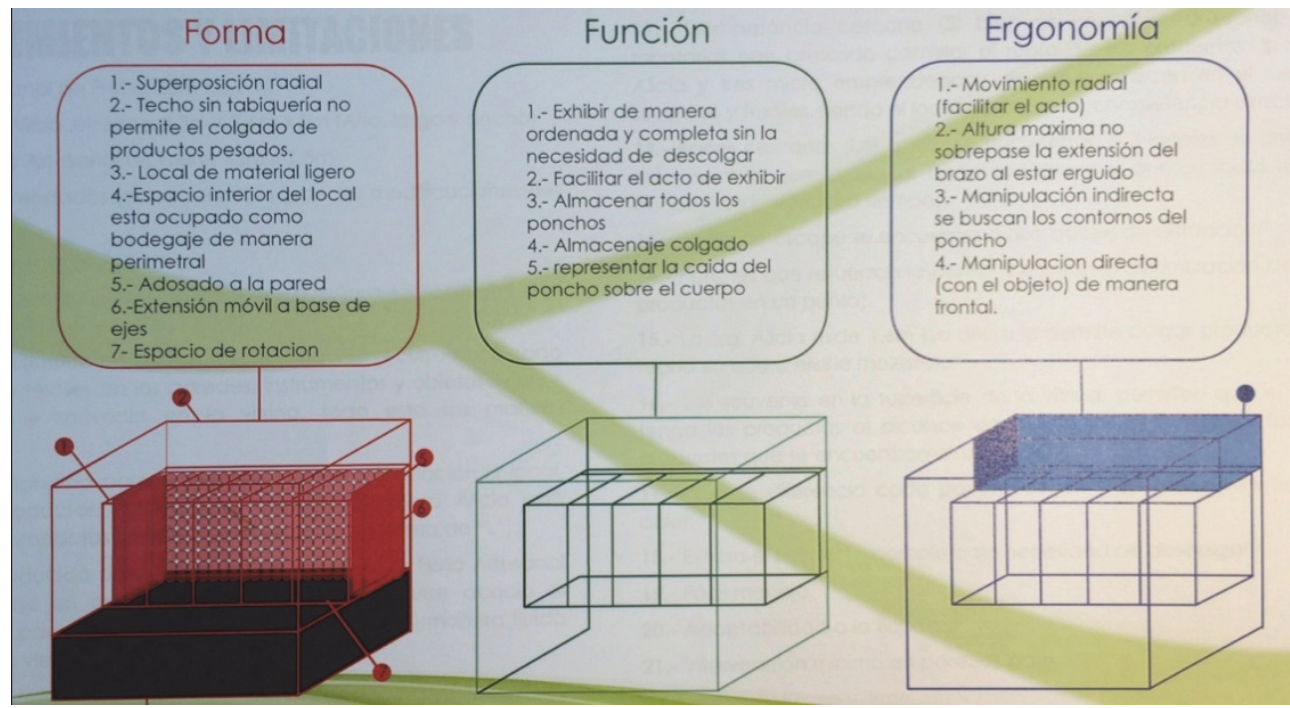

Fig. 3 Modelos Conceptuales. Fuente: Maureen Muñoz,Carmen Pereira y Melissa Quezada (2013).

\subsection{Integración del espacio del diseño}

El proceso se inicia valorando cada atributos de cada subsistema (forma,función y ergonomía) y las correlaciones que entre estos pueda existir. Junto con eso se esquematiza la integración en una 
representación análoga del espacio del diseño donde se van representando las geometrías principales de los atributos seleccionados.

En este caso particular, como siguiente etapa los estudiantes tuvieron que construir una maqueta del espacio del diseño. Esta maqueta en baja fidelidad buscaba representar físicamente las geometrías representantes de los atributos fundamentales del producto: Volúmenes, Superficies y limites de contornos de la forma, función y ergonomía). Este insumo tangible, les facilitó la identificación de los atributos y de sus posibles consecuencias y efectos dentro de la propuesta de diseño.

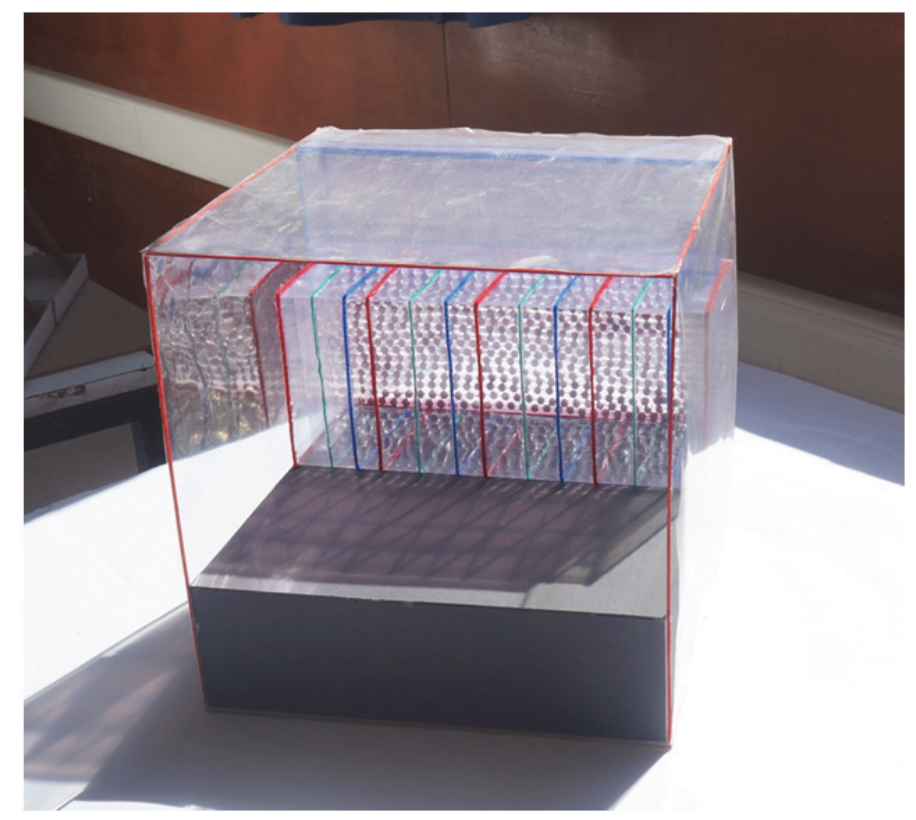

Fig. 4 Maqueta Espacio del Diseño Fuente: Maureen Muñoz,Carmen Pereira y Melissa Quezada (2013).

\subsection{Desarrollo Formal y Propuesta Final}

Considerando la modelación del espacio del diseño así como los objetivos y requerimientos se procedió a explorar las posibles formas y configuraciones del producto tal como se puede observar en las figuras $5 \mathrm{y}$ 6. Considerando la opinión y retroalimentación del usuario dentro de la elección, desarrollo y refinamiento de la propuesta de diseño.

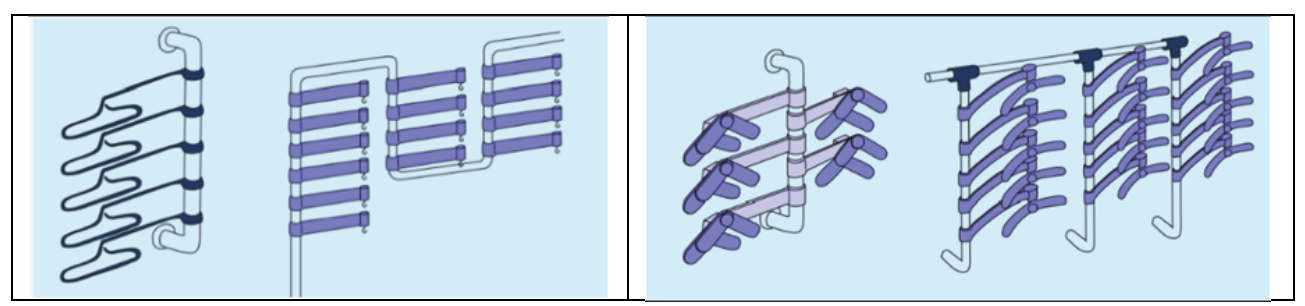

Fig. 5 Desarrollo Formal. Fuente: Maureen Muñoz, Carmen Pereira y Melissa Quezada (2013). 


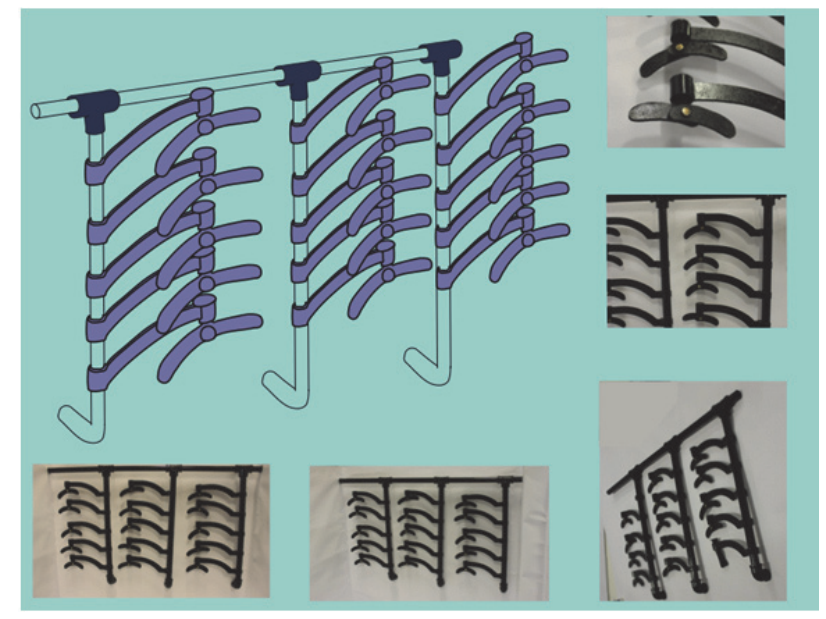

Figura 6. Prototipo de propuesta de diseño. Fuente: Maureen Muñoz ,Carmen Pereira y Melissa Quezada (2013).

\subsection{Estudio}

Para caracterizar los resultados de la implementación del enfoque sistémico en la etapa de diseño conceptual se realizó un estudio de alcance descriptivo, con una orientación exploratoria, mediante un análisis pre-experimental. Para recolectar los datos se aplicaron diferentes encuestas al final del taller, donde se evaluó la percepción de los estudiantes participantes respecto a la aplicación del enfoque sistémico en el diseño y desarrollo de productos.

La encuesta fue aplicada a 15 estudiantes de 3er año de la carrera de Diseño Industrial de la Universidad del Bío Bío, la única universidad estatal de la ciudad de Concepción, Chile. Estos alumnos cursaban el taller [DCU] como parte de sus asignaturas obligatorias. Fueron seleccionados mediante muestreo no probabilístico por accesibilidad y representan el 54,3\% de los estudiantes que cursaron la asignatura.

El cuestionario aplicado en el proceso de encuesta incluía dos partes, una centrada en la percepción de la metodología sistémica y su utilidad en el proceso de diseño, y la segunda referida a la utilidad específica de dos herramientas empleadas durante la actividad: la representación de atributo en forma de geometrías y la construcción de una maqueta tridimensional. La encuesta presentaba nueve preguntas, seis de la primera parte y tres de la segunda parte del cuestionario, exhibiendo afirmaciones sobre el funcionamiento de la actividad. Para responder, los estudiantes debían emplear una escala Likert de cinco alternativas (1: Muy en desacuerdo; 2: En desacuerdo; 3:Indiferente; 4: De acuerdo; 5: Muy de acuerdo), según su percepción sobre lo vivenciado, Tabla 2. El cuestionario pasó previamente por juicio de expertos. 


\begin{tabular}{|c|c|c|c|c|c|}
\hline & 1 & 2 & 3 & 4 & 5 \\
\hline $\begin{array}{l}\text { 1. Aplicar la metodología sistémica me facilitó el proceso de análisis } \\
\text { del producto a diseñar. }\end{array}$ & & & & & \\
\hline $\begin{array}{l}\text { 2. El análisis desagregado de las variables en forma, función y } \\
\text { ergonomía me permitió identificar aspectos claves del producto. }\end{array}$ & & & & & \\
\hline $\begin{array}{l}\text { 3. La metodología sistémica me permitió llevar un registro del } \\
\text { proceso que permita fundamentar las decisiones. }\end{array}$ & & & & & \\
\hline $\begin{array}{l}\text { 4. El modelo sistémico me ofreció una referencia teórica con los } \\
\text { requerimientos y aspectos a tener en consideración al momento de } \\
\text { generar la propuesta de diseño formal }\end{array}$ & & & & & \\
\hline $\begin{array}{l}\text { 5. El análisis me permitió diferenciar los atributos o variables } \\
\text { asociados a forma, función y ergonomía }\end{array}$ & & & & & \\
\hline $\begin{array}{l}\text { 6. La metodología sistémica me ayudo a comprender mejor el } \\
\text { problema }\end{array}$ & & & & & \\
\hline Representación del Espacio del Diseño: & & & & & \\
\hline $\begin{array}{l}\text { 1. La representación de los atributos en forma de geometrías me } \\
\text { permitieron comprender la relación entre los atributos identificados } \\
\text { dentro del espacio. }\end{array}$ & & & & & \\
\hline $\begin{array}{l}\text { 2. Construir la maqueta tridimensional me permitió identificar la } \\
\text { implicancia en el contexto real de los atributos estudiados. }\end{array}$ & & & & & \\
\hline $\begin{array}{l}\text { 3. La maqueta del espacio de diseño fue un insumo que facilitó la } \\
\text { comunicación y colaboración con los miembros del equipo. }\end{array}$ & & & & & \\
\hline
\end{tabular}

\section{Resultados}

En el siguiente apartado se exhiben los resultados obtenidos a partir de las opiniones y percepciones de los estudiantes con respecto a la contribución del uso de la metodología sistemática y la realización de la maqueta del espacio del diseño en la solución final de diseño.

A continuación se detallan los resultados según los siguientes apartados:

\subsection{Evaluación global de la metodología sistémica:}

En cuanto al uso de la metodología sistémica, un 40\% de los estudiantes está de acuerdo con que aplicar la metodología sistémica facilita el proceso de análisis del producto a diseñar, seguido por un $33 \%$ que está muy de acuerdo con la aplicación de la metodología sistémica, Figura 7. 


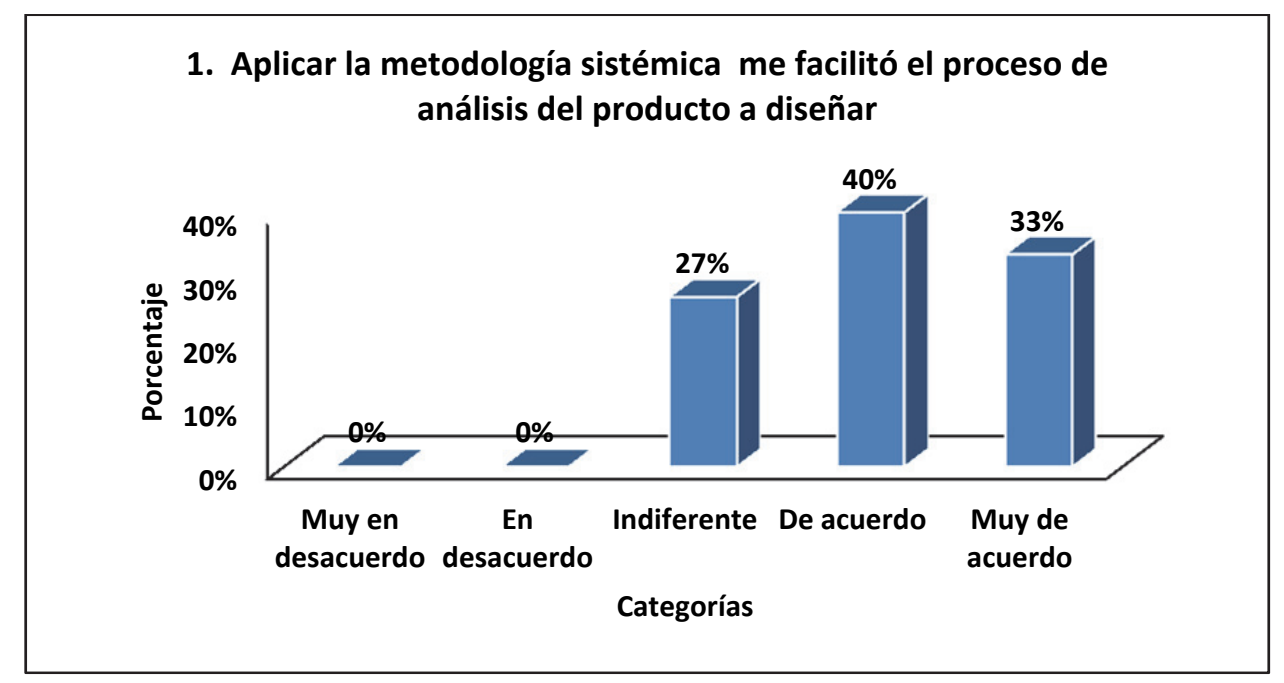

Figura 7. Evaluación de la utilidad de la metodología sistémica para facilitar el proceso de análisis del producto a diseñar.

Por otro lado, un $53 \%$ de los encuestados está muy de acuerdo con que el análisis de las variables en forma, función y ergonomía permite identificar aspectos claves del producto. Sin embargo, un 13\% de los encuestados se reportó indiferente y un 7\% se considera en desacuerdo, Figura 8.

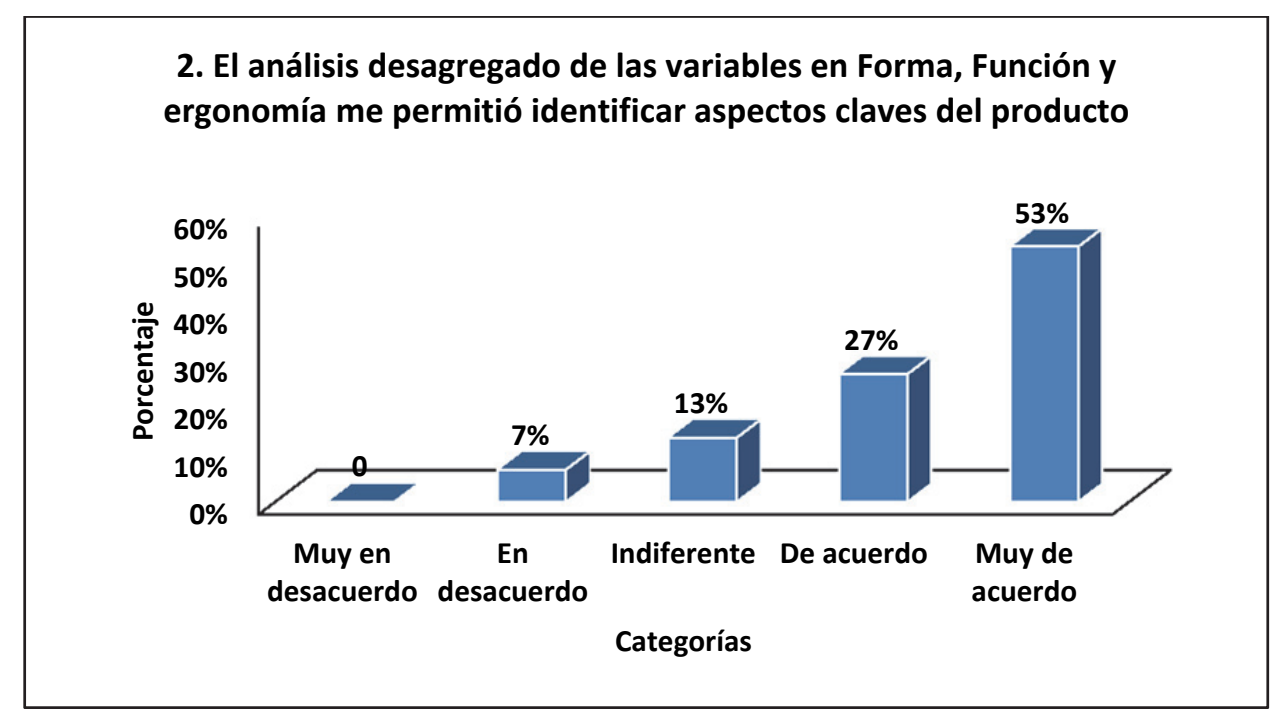

Figura 8. Evaluación de la utilidad del análisis para identificar aspectos clave del producto diseñado.

En cuanto a si la metodología sistemática permite registrar el proceso de cara a una toma de decisiones fundamentadas, un $74 \%$ de las respuestas de los ex alumnos del curso se encuentra en las categorías de acuerdo o muy de acuerdo, Figura 9. 


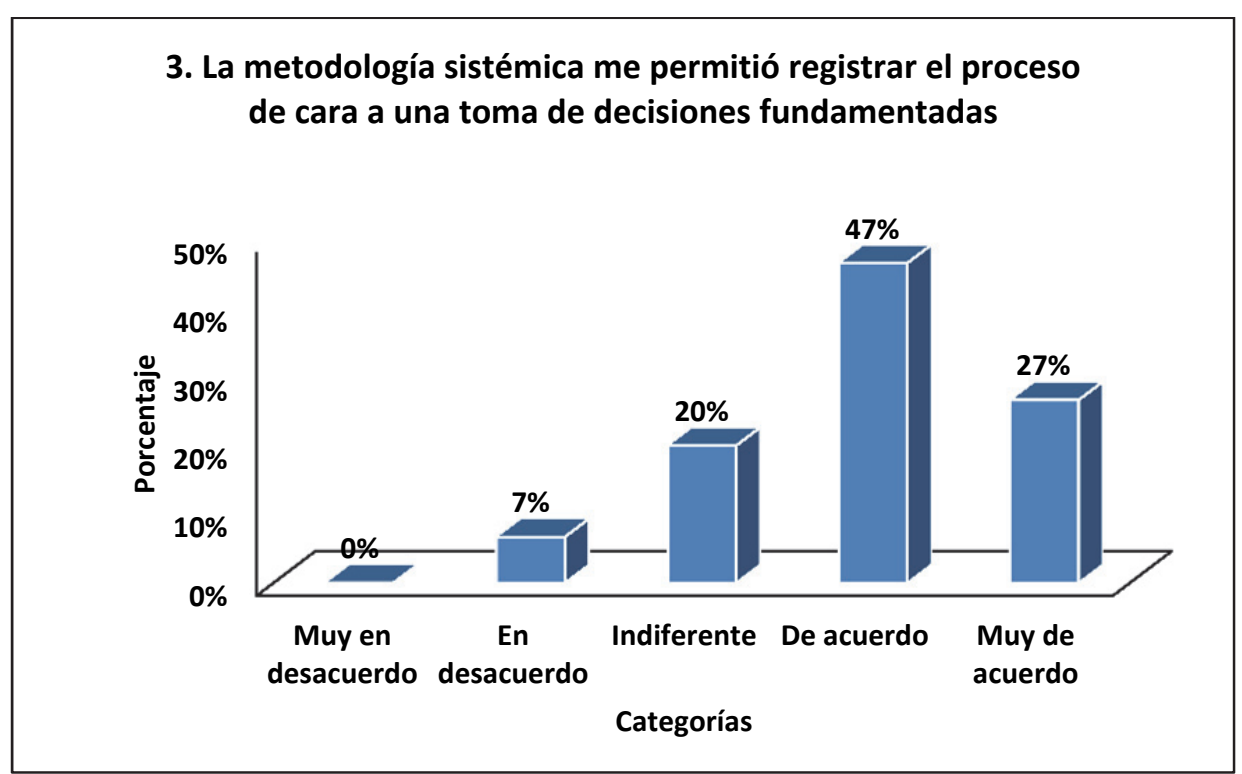

Figura 9. Evaluación de la utilidad de la metodología sisémica para registrar el proceso y fundamentar la toma de decisiones.

El 83\% de los estudiantes está de acuerdo o muy de acuerdo en que el modelo sistemático ofrece una referencia teórica con los requerimientos y aspectos a tener en consideración al momento de generar el diseño conceptual, Figura 10.

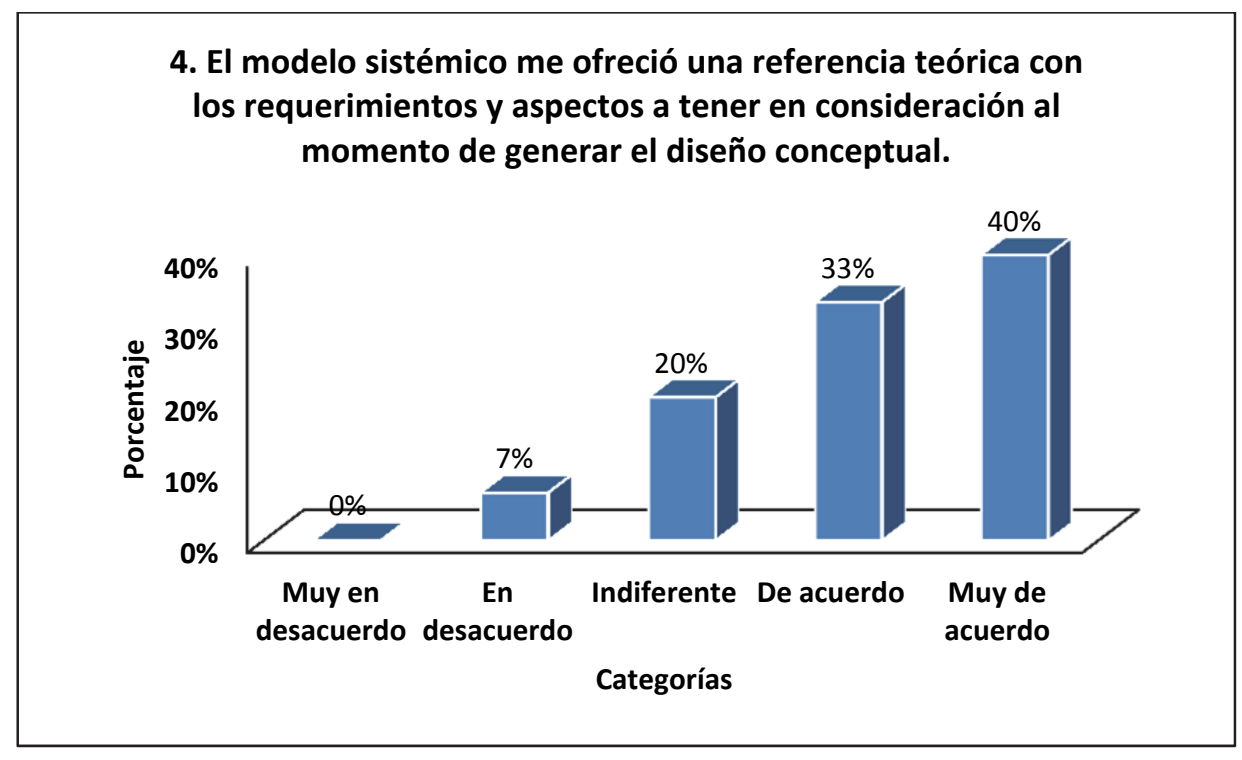

Figura 10. Evaluación de la utilidad de la metodología sistémica para ofrecer una referencia teórica.

El 69\% de los encuestados encuentra que el análisis permite diferenciar los atributos o variables asociadas a forma, función y ergonomía y un 15\% que esta acuerdo con esta afirmación, Figura 11. 


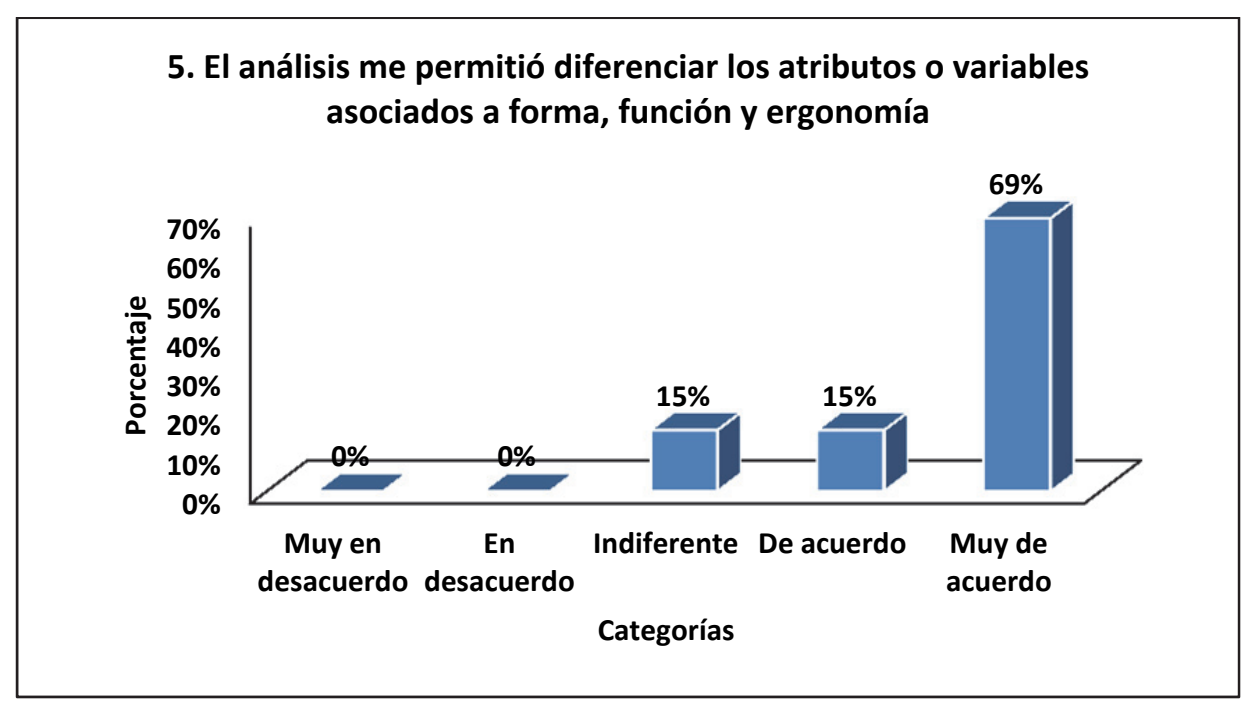

Figura 11. Evaluación de la utilidad de la metodología sistémica para diferenciar atributos asociados a forma, función y ergonomía.

Un $69 \%$ de las respuestas de quienes cursaron la asignatura estuvieron de acuerdo o muy de acuerdo en que la metodología sistemática ayuda a comprender mejor el problema, Figura 12.

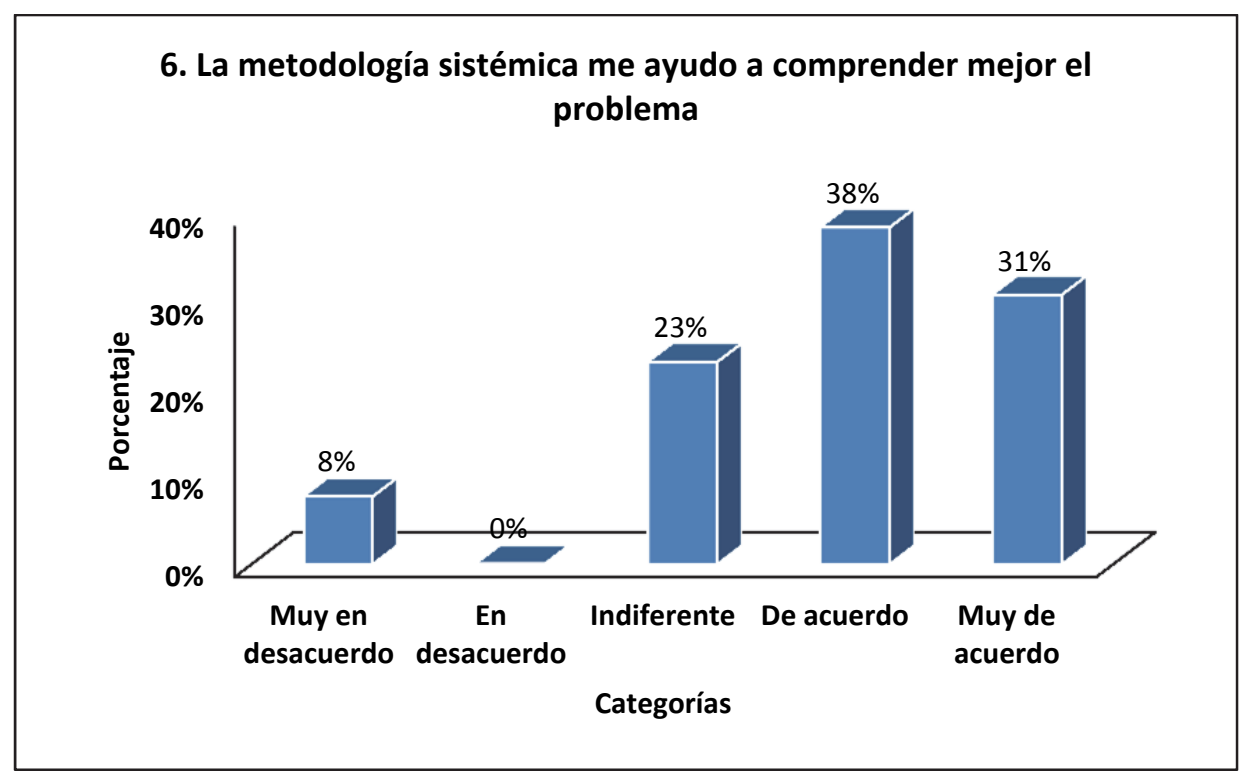

Figura 12. Evaluación de la utilidad de la metodología sistémica para comprender mejor el problema.

\subsection{Evaluación de herramientas específicas.}

La segunda parte del cuestionario estuvo referida a la percepción de herramientas específicas: puntualmente el uso de representación de geometrías para comprender la relación entre atributos identificados dentro del espacio; el empleo de maquetas tridimensionales para identificar la implicancia de los atributos empleados; el uso de la maqueda del espacio de diseño como insumo para facilitar la 
comunicación y la colaboración, y su aporte para explorar diversos modos y configuraciones de los atributos.

En el primer caso, un $73 \%$ de los estudiantes estuvo muy de acuerdo o de acuerdo con que la representación de los atributos en forma de geometrías permite comprender la relación entre los atributos identificados dentro del espacio, Figura 13.

Representación del Espacio de Diseño

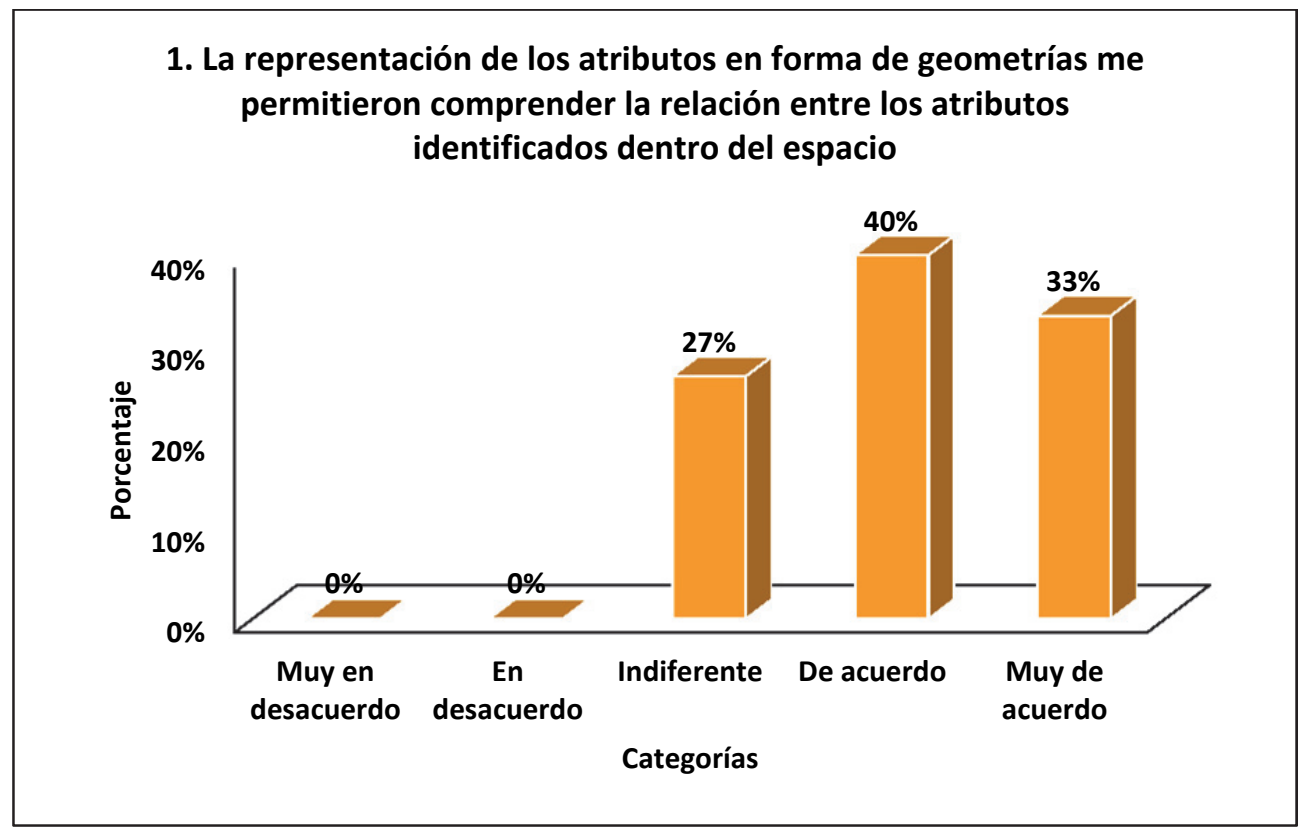

Figura 13. Evaluación de la utilidad del uso de formas de geometría para comprender la relación entre atributos identificados.

En la segunda agirmación, un $83 \%$ de los ex alumnos se mostró de acuerdo o muy de acuerdo en que construir la maqueta tridimensional permite identificar la implicancia en el contexto real de los atributos estudiados, Figura 14. 


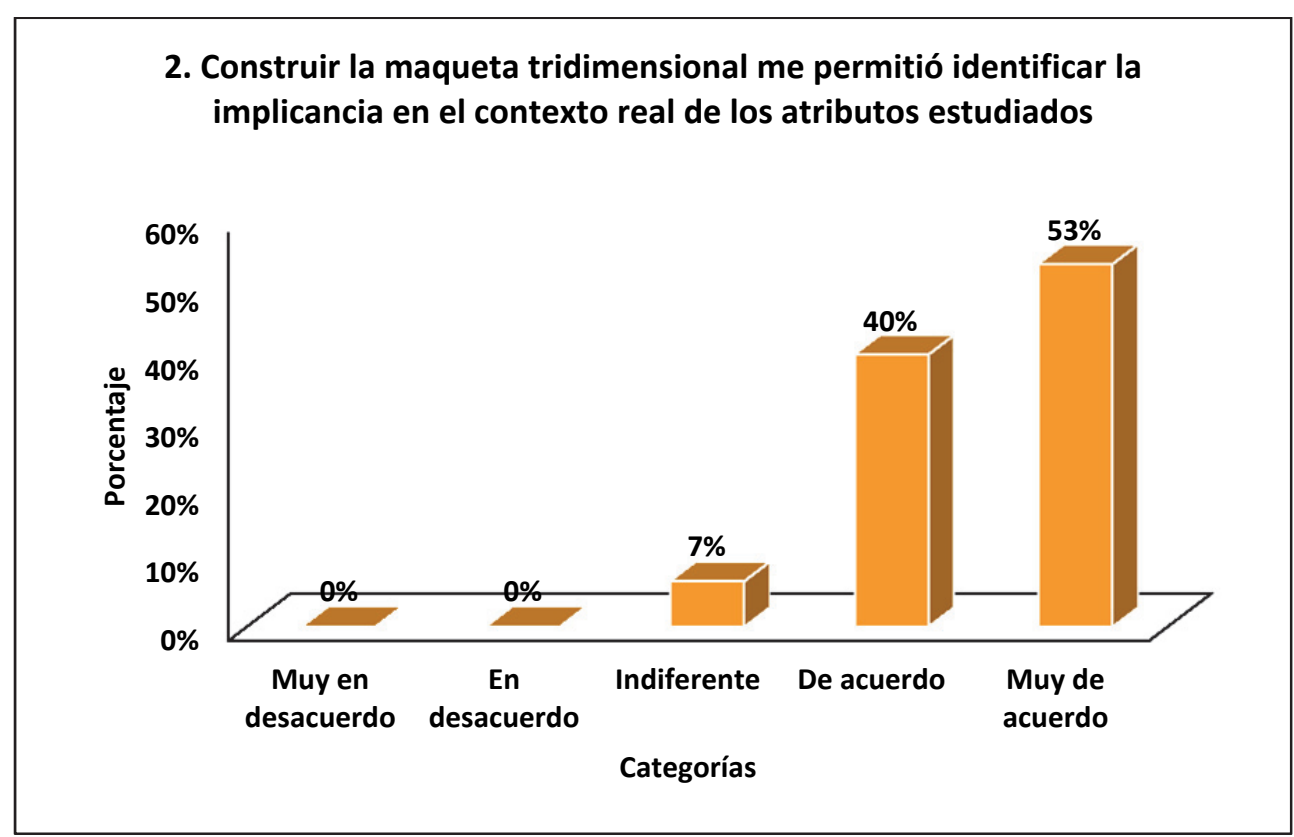

Figura 14. Evaluación de la utilidad de la maqueta tridimensional para identificar la implicancia de los atributos en el contexto.

Y finalmente, un 67\% de los jóvenes está de acuerdo o muy de acuerdo en que la maqueta del espacio de diseño fue un insumo que facilitó la comunicación y colaboración con los miembros del equipo, aunque $33 \%$ se mostró indiferente ante esta afirmación, Figura 15

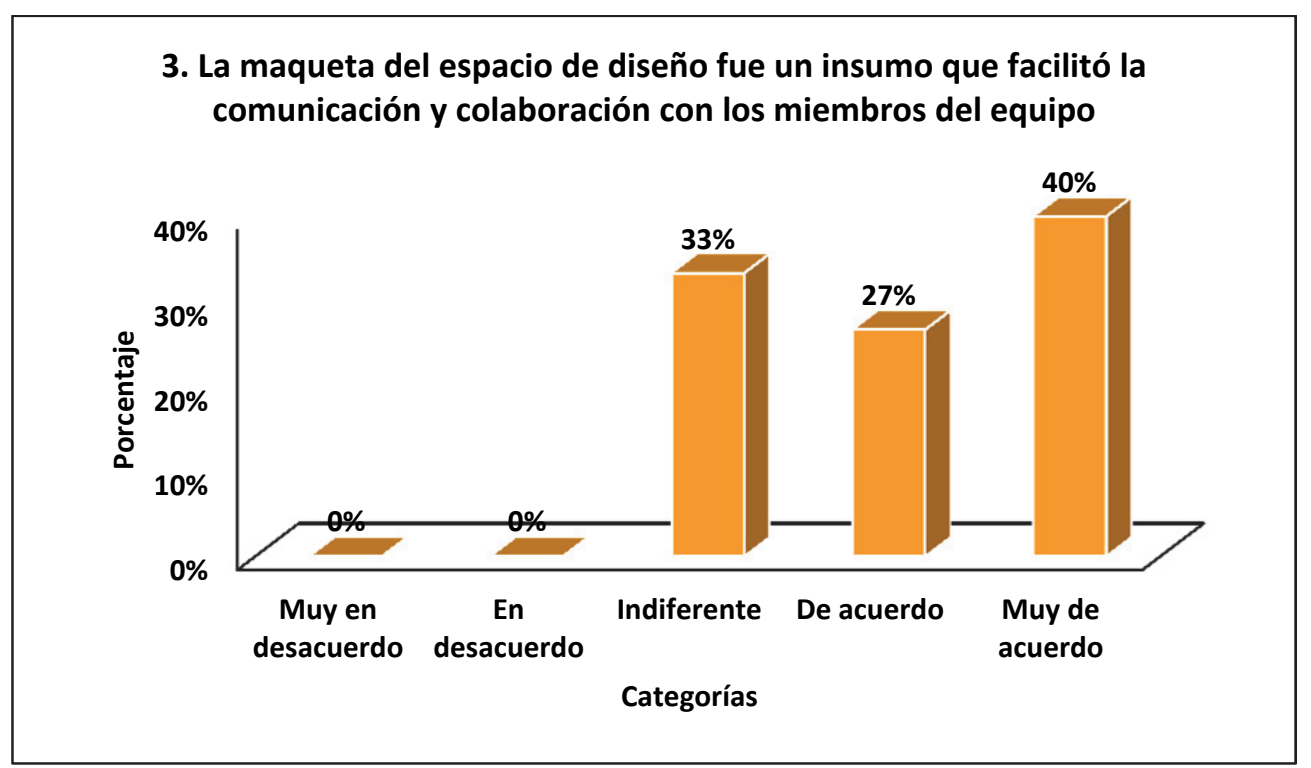

Figura 15. Evaluación de la utilidad de la maqueta tridimensional para facilitar la comunicación y colaboración dentro del equipo.

A la luz de los resultados y a la experiencia de dicha actividad, se plantea en la siguiente figura la secuencia de la desagregacion sistémica y su relación con las representaciones de diseño.Evidenciando 
los tipos de representaciones que se utilizan en cada una de sus fases. Considerando la construcción de la maqueta en baja fidelidad dentro de la exploracion y definición del espacio del diseño.

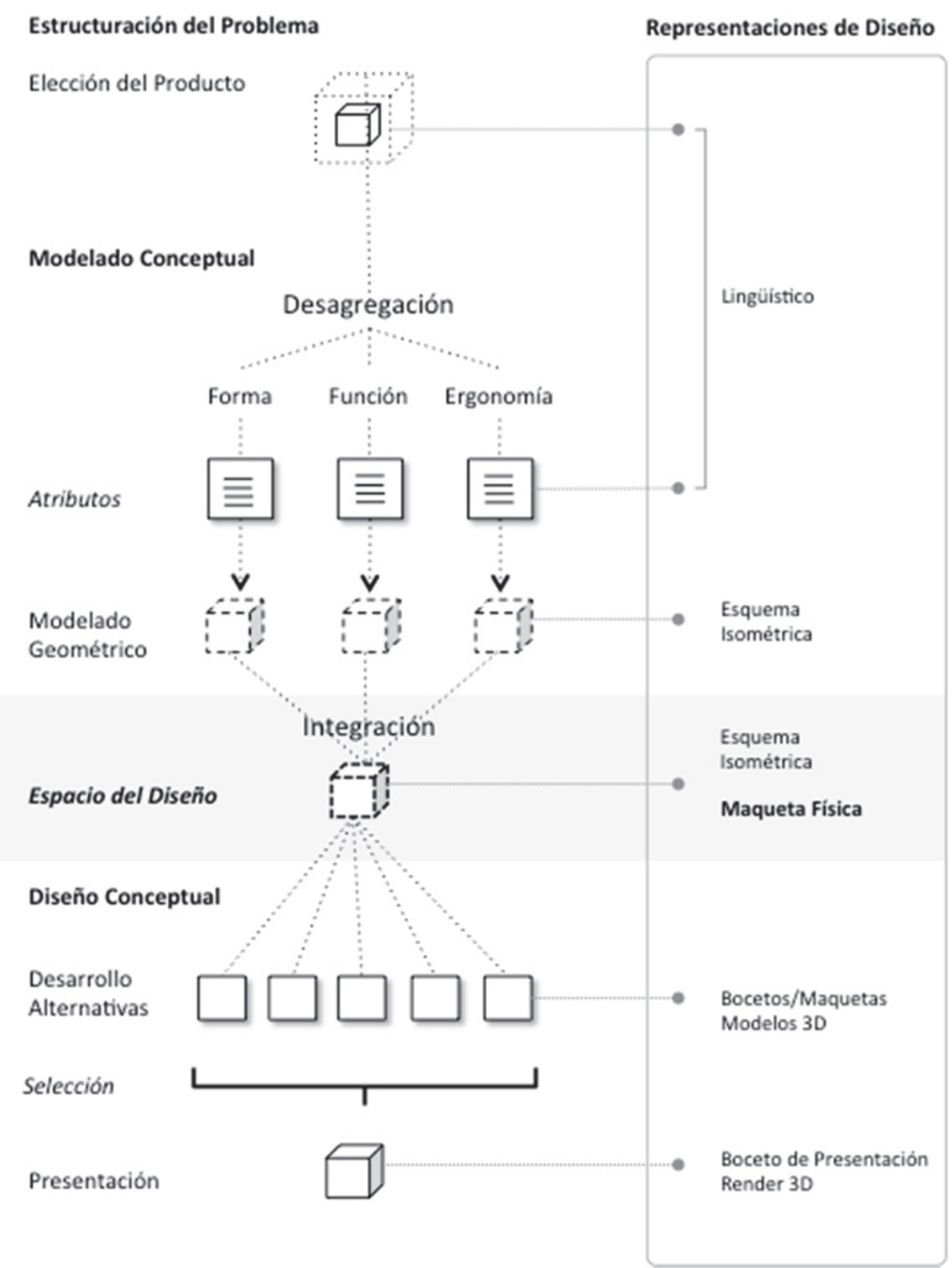

Fig. 16 Secuencia Aplicación Modelado Sistémico. Fuente: Elaboración Propia basada en Briede et al. 2014

\section{Conclusiones}

La metodología sistémica, permite el análisis detallado de tres variables fundamentales de un producto de diseño (forma, función y ergonomía), que al separarlos facilita notoriamente la evaluación y jerarquización de sus condicionantes, relacionándolas además, con el potencial comportamiento que el usuario espera del objeto en cuestión, esto aparece claramente reconocido por la mayoría de los estudiantes que participaron de la experiencia.

Pero también en paralelo, otra ventaja es que establece los criterios teóricos o experienciales sobre los que se fundamentan dichas jerarquizaciones, lo que racionaliza su puesta en valor.

Por otra parte, la representación del Espacio de Diseño permite relacionar dichas jerarquías y argumentos a su dimensión tangible, concentrando la atención del diseñador en aquellos conceptos relevantes, 
evidenciando de esta manera aquellas variables del diseño formal que serán coherentes con los conceptos y su respectiva argumentación lógica de valor que sobre lo esperado del producto. Este último punto resulta ser uno de los más importantes de la propuesta metodológica, porque verbalizar un discurso en relación a su formalidad de manera coherente, implica una internalización clave y esencial que demuestra la transferencia de un conocimiento abstracto a una apuesta tangible y fundamentada. Esta transferencia será aplicable al mundo laboral, como una estrategia argumentativa que el diseñador requerirá siempre, ya sea para ofrecer su producto, como para resolver en distintos ámbitos, las soluciones más apropiadas a las diversas problemáticas y contextos.

Finalmente, en cuanto a las herramientas específicas, tanto las representaciones geométricas como la maqueta fueron muy bien evaluadas, aunque un $27 \%$ se reportó indiferente al impacto de las primeras en comprender los atributos identificados dentro del espacio y un 33\% pensó lo mismo en cuanto a la utilidad de las maquetas para favorecer la comunicación y colaboración. Pese a esto, un $93 \%$ de los encuestados consideró que éstas últimas permitían identificar la implicancia de los atributos en el contexto real, por lo que demuestran su utilidad para estos fines, llevando a cuestionarse de qué forma podría potenciarse también su utilidad comunicativa.

Pese a esto, existe un grupo minoritarío de estudiantes que mostraron su desacuerdo con que la metodología ayudaba a identificar aspectos clave del producto, permitía el registro del proceso, aportaba una referencia teórica y permitía comprender mejor el problema. Si bien estos alumnos nunca representaban más de un $8 \%$ cabe cuestionarse si se debe a estudiantes que no lograron realizar una adecuada implementación de estas herramientas o, por el contrario, identificaron debilidades en la implementación de la misma. Por este motivo, para futuras instancias, se sugiere realizar integrar herramientas cualitativas como entrevistas o grupos focales que permitan obtener una visión más experiencial y procesual de la implementación de estas herramientas.

\section{Agradecimientos}

Los autores desean agradecer a la Dirección de Investigación de la Universidad del Bío-Bío y su fondo FAPEI.

\section{References}

BALlerini, A., CASTRO F., CONTRERAS W., SALAZAR M., LAZO C., LETELIER P.VÁZQUEZ G., CÁCERES H.VIDAL J.,RAMIS F.,ARANEDA G., PARRA J.C.,HASSE V.,VALENZUELA H.,CORRALES J.,GÓMEZ G., SAN JUAN L., VALASSINA F., LASO P., GRANDÓN E. \& CIFUENTES M.T. (2009) Modelo Educativo de la Universidad del Bio-Bio. Comisión de renovación curricular, Vicerrectoría académica 2008, Universidad del Bío-Bío, Ediciones Universidad del Bío-Bío.

BARBERÀ, E.; MAS, X.; GUÀRDIA, L \& VALL-LLOVERA, M. (2008) "El e-transfolio: la evaluación de competencias desde una perspectiva de aprendizaje. 2.0.," V. Congreso Iberoamericano de Docencia Universitaria (CIDU), Valencia.

BRIEDE , J.C.; CARTES, J.; BUSTAMANTE, A. \& PEREZ, M. (2011) "Shaping the individual designer: participatory design in emergency context," Proceedings of the 18th International Conference on Engineering Design ICED11, Copenhagen Denmark, 15-18 Aug. vol.8, 173-183.

BRIEDE, J.C., CABELLO, M. \& HERNANDIS, B. (2014) Modelo de abocetado concurrente para el diseño conceptual de productos industriales, Dyna, 81(187) 199-208

CHAUNCEY, W., (2010) Handbook of User-Centred Design Methods. USA: Morgan Kaufmann. USDE, U.S. Department of Education, National Center for Education Statistics (2001) Defining and Assessing Learning: Exploring Competency-Based Initiatives, Washington, D.C., USA. 
CRESWELL, J. (2003) "Research Design, Qualitative, Quantitative and Mixed Methods Approaches". Sage Publications SA.

DYM, C., AGOGINO, A., ERIS, O., FREY, D. \& LEIFER, L. (2005) Engineering Design Thinking, Teaching, and Learning. Journal of Engineering Education, 103-120.

HERNANDIS, B. \& BRIEDE, J. (2009) An educational application for a product design and engineering systems using integrated conceptual models. Ingeniare. Revista chilena de ingeniería, N17(3) 432-442

KRIPPENDORF K. (2006) The semantic turn: a new foundation for design. Boca Raton: CRC/Taylor \& Francis Group.

LIM, Z. , ANDERSON, C. \& MCGRATH, S. (2012) "Professional Skills Development in a Resource- Poor Setting: The Case of Pharmacy in Malawi," International Journal of Educational Development vol.32, no. 5, 654-664.

MABARDI J.F.(2012)Maestría del Proyecto. Apuntes para la práctica de la enseñanza del proyecto.Ediciones Universidad del Bío-Bío. Concepción. Chile.

MOYANO, E., CASTILLO, R. \& LIZANA, J. (2008). Trabajo informal: motivos, bienestar subjetivo, salud, y felicidad en vendedores ambulantes. Psicologia em Estudo, 13(4), 693-701.

SCHÖN, D.A. (1983) The Reflective Practitioner: How Professionals Think in Action, New York: Harper Collins.

VOORHEES RICHARD A. (2001) “Competency-Based Learning Models: A Necessary Future,” New Directions for Institutional Research 110, 5-13.

WALTER D. (2000) "Competency-based on-the-job training for aviation maintenance and inspection - a human factors approach,” Int. J. of Ind. Ergonomics 26, 249-259. 